\title{
Grundsätze christlicher Redaktionsarbeit in Afrika
}

\author{
von Ezekiel C. Makunike
}

Was sind "redaktionelle Richtlinien" oder Richtlinien überhaupt? Sie können definiert werden als "Leitkurs oder Richtung für die Leitung einer Zeitung". Sie haben zu tun mit der Kontinuität der Blickrichtung wie der Publikationspraxis. Sie sind ein Kurs, dem sich die Zeitung verpflichtet weiß in Beantwortung der beiden überaus wichtigen Fragen: "Was sollen wir drucken?" und "Wie sollen wir es drucken?"

Die Richtlinien bestimmen Praxis, Regeln und Prinzipien, die sich die Zeitung selbst als Standard und Maß setzt. Insofern regeln sie jede Phase der Nachrichtenverarbeitung, die ideologische Richtung und sogar die Größe und das Aussehen der Drucktypen.

Die Zeit, die zur Formulierung eines guten und klaren Redaktionskonzepts verwendet wird, ist gut angelegt. Sie erspart dem Redakteur und seinen Mitarbeitern viele Unsicherheiten und schützt vor unklaren und verschwommenen Darstellungen. Ein gutes Redaktionskonzept wird die schwierige Aufgabe der Inhaltswahl, also Annahme oder Ablehnung in einer bestimmten Ausgabe der Zeitung, erleichtern.

Was hier gesagt wird, gilt für kirchliche und weltliche Periodika in gleicher Weise. Meine Aufgabe hier beschränkt sich auf die Beschreibung solcher Richtlinien für kirchliche Zeitschriften und Zeitungen im heutigen Afrika. Glücklicherweise brauche ich nicht die Notwendigkeit kirchlicher Zeitschriften in Afrika heute nachzuweisen. Vorausgesetzt wird, daß der kirchlichen Presse im heutigen Afrika eine wichtige Rolle zufällt. Ich brauche das nicht eigens zu betonen.

In vielen Teilen Afrikas haben die Kirchen die ersten Periodika gegründet; mancherorts stehen sie auch heute noch mit dieser Aufgabe ganz allein. Sie informieren die örtliche Bevölkerung, sie leisteten ihren Beitrag bei der Festlegung der Orthographie der lokalen Sprachen und gaben manchmal einheimischen Autoren die Startmöglichkeiten für ihre Karriere im gedruckten Wort.

Aber heute bestehen in den meisten Teilen Afrikas die kirchlichen Zeitschriften neben einer großen Zahl anderer Zeitungen und Magazine. Die christlichen Zeitschriften haben jetzt Partner und - ehrlich gesagt - stärkere Partner bekommen, was Ausbildung, Finanzen, Leserinteresse und Gesamteinfluß angeht.

Es stellt sich daher die Frage:

„Sollen christliche Periodika ihren eigenen Wirkungsbereich suchen, oder sollten sie versuchen, mit der weltlichen Presse zu konkurrieren, weil sie, die christlichen Zeitschriften, hier die Pionierarbeit geleistet haben?"

Ezekiel C. Makunike ist Direktor des Africa Literature Centre der Okumenischen Mindolo Stiftung in Kitwe, Sambia. 
Dies ist die grundlegende Frage bei der Erstellung von Richtlinien.

In der schwierigen Finanzsituation unserer Zeit ergibt sich folgende Fragestellung:

-Kann die kirchliche Presse möglicherweise Schrittmacher sein für die Zeitgenossen, ader sollte sie sich mit einem zweiten Platz zufriedengeben, indem sie nur Einzelfragen aufgreift oder die übriggebliebenen Stücke sammelt? ${ }^{\alpha}$

Ich bin fest überzeugt, daß die kirchliche Presse im heutigen Afrika eine Aufgabe hat und eine solche $\mathrm{zweite}$ Rolle nicht zu spielen braucht. Wenn auch die Stimme der Kirche heute überall in der Welt zurückgedrängt scheint, wird sie doch noch immer als die Stimme des Gewissens, als eine Stimme menschlicher Sorge verstanden.

Besonders in Afrika, wo traditionell der Mensch im Mittelpunkt aller Bemühungen steht, ist einer Institution, die sich um die Förderung und Weiterentwicklung des Menschen bemüht, ein Ehrenplatz sicher. Die Kirche steht hier eindeutig an der Spitze aller anderen Institutionen mit ähnlichen Zielen. Leider ist aber auch eine Folge solcher Bemühung, daß die Kirche allzu selbstzufrieden geworden ist. Sie war ihrer selbst so sicher, daß sie die Anpassung an die Anforderungen unserer Zeit zu langsam vollzogen hat.

Wie wir gesehen haben, ist die Kirche auf dem Gebiet der Presse nicht unerfahren. Häufig hört man, daß kirchliche Hospitäler der Offentlichkeit einen besseren Dienst erweisen, daß kirchliche Schulen bessere Ergebnisse erzielen als andere. Doch kaum je ist zu vernehmen, daß kirchliche Zeitungen besser seien als andere. Woran liegt das? Sicher nicht nur an den fehlenden Geldern, wie wir oft glauben. Krankenhäuser und Schulen müssen sich ebenso nach der Decke strecken. Der Grund könnte vielmehr darin liegen, daß wir im Bereich der Presse zu unüberlegt und zufällig ans Werk gehen. Krankenhäuser und Schulen sind besser, weil Ärzte, Schwestern, Lehrer und Schulleiter hervorragend ausgebildet werden. Zudem gibt es eine ständige strenge Aufsicht über die Einhaltung der geforderten Maßstäbe. Von einigen Ausnahmefallen abgesehen, sind die Herausgeber kirchlicher Periodika mit anderen Arbeiten berlastet, manchmal in einer nicht zumutbaren Weise; für die Ausbildung und Weiterbildung von Redakteuren gibt es keine festen Richtlinien. Die Möglichkeiten zur Weiterbildung sind rechtlich nicht abgesichert. Es gibt keine beruflichen Klubs oder Vereinigungen für kirchliche Zeitungsleute, die zudem - aus welchem Grunde wuch immer - von weltlichen Journalisten nicht als gleichgestellte Partner akzeptiert trerden.

Leider wird nicht immer gesehen, daß kirchliche Publikationen gegenüber weltlichen Zeitungen viele Vorteile haben. Sie brauchen nicht die abgestandenen Nachrichten zu wiederholen, die schon in der Tagespresse veröffentlicht wurden, sondern haben mehr Zeit, um für ihre Darlegungen mehr Fakten und Hintergrundmaterial zu mmeln. Das macht eine größere Gründlichkeit und bessere Kommentierung mögHich. Auf diese Weise gewinnt der Leser zu seiner Zeitung Vertrauen, weil er die Hintergründe hinter den Nachrichten besser versteht. So wird auch der Luxus einer besseren, d. h. gefälligeren und ansprechenderen Aufmachung möglich. In vielen Dällen könnte auch die Reaktion des Lesers gezielt auf bestimmte Inhalte gelenkt rerden, die in der Tagespresse erscheinen. So wäre die kirchliche Presse eine Ergänzung und ein Stimulans für die Tageszeitungen. 


\section{Grundlagen für redaktionelle Richtlinien}

Gute Richtlinien ergeben sich aus klar konzipierten Zielen, die die Existenz eines Periodikums rechtfertigen. Solche Richtlinien müssen Antwort auf die folgenden Fragen geben:

a) Warum haben wir dieses Periodikum?

b) Was wollen wir erreichen?

c) Worauf wollen wir besonderen Wert legen?

d) Soll es eine Meinungszeitung sein, eine Publikation mit pädagogischen Zielen für Frauen, Jugendliche, oder eine allgemeine Zeitung?

e) In welchem Verhältnis sollen raummäßig Anzeigen und Presseinformation stehen; welche Anzeigen nehmen wir an und welche lehnen wir ab?

f) Eine sehr wichtige Frage ist auch:

Welches sind die von unseren Leuten empfundenen Fragen und Bedürfnisse, zu denen wir einen Beitrag leisten können? Solche Bedürfnisse könnten sein: nationale Einheit, nationale Entwicklung, Stimme für die Stimmlosen oder Machtlosen, Kampf gegen negative Auswirkungen des Stammesdenkens (tribalism), Kampf gegen Rassismus und Unterdrückung.

Für ein Kirchenblatt ist die Entscheidung darüber wichtig, wieviel Raum rein kirchlichen Nachrichten und wieviel allgemeinen und nationalen Nachrichten gegeben werden sollte. Auch ist es wichtig zu entscheiden, wieweit die Zeitung politische Fragen initiieren oder kommentieren sollte.

\section{Welche Faktoren beeinflussen die redaktionellen Ricbtlinien?}

Es gibt deren viele, u. a.:

a) kirchliche oder konfessionelle Glaubensbekenntnisse,

b) politische Stimmung im Land,

c) Art der Finanzierung: Unterstützung oder Anzeigengelder,

d) Qualifizierung des Herausgebers und seiner Mitarbeiter,

e) Mentalität der Menschen nach Gebiet und Zeitpunkt.

Zweck dieses Beitrags ist nicht, Lösungen anzubieten, sondern die grundsätzlichen Probleme und Fragen klar darzulegen, die die Situation der Kirchenpresse im heutigen Afrika bestimmen. Wir hoffen, durch Erfahrungsaustausch gemeinsam Lösungen für diese gemeinsamen Probleme zu finden.

\section{Besondere Probleme eines kirchlichen Redakteurs in Afrika}

Leider befindet sich der Redakteur einer christlichen Zeitung in Afrika heute manchmal in einer nicht beneidenswerten Situation. Er steht zwischen den Fronten: auf der einen Seite steht die Macht westlicher Werte, die ihrerseits Maßstäbe setzen möchten, auf der anderen Seite die Betonung der afrikanischen Werte. Da gewöhnlich die Finanzierungsmodelle der meisten Kirchenzeitungen von westlichen Institutionen abhängig sind, findet sich der Redakteur einer Kirchenzeitung in Afrika hinund hergeschoben zwischen westlichen und afrikanischen Werten und den politischen Gefühlèn und Wünschen der Bevölkerung. 
Viele afrikanische Länder haben zum Beispiel herausgefunden, daß auf der jetzigen Stufe ihrer Entwicklung Vielparteienregierungen ein teurer Luxus sind. Das gilt auch für Vorstellungen wie die Gegenüberstellung von afrikanischem Sozialismus und westlichem Kapitalismus, sambischem Humanismus und der Philosophie der Authentizität in Zaïre. Doch die meisten Mitarbeiter aus dem Westen sind nicht bereit, diese Sicht der Dinge zu akzeptieren. Sie beharren, verständlicherweise, auf ihrer Sicht, die wiederum auf ihrem System beruht.

Bei der Betonung der kirchlichen Beteiligung an der nationalen Entwicklung in unseren Tagen darf erwartet werden, daß die meisten Redakteure christlicher Zeitungen, wie ihre Mitbürger, Mitglieder der nationalen politischen Parteien sind. Daran ist nichts Falsches oder Außergewöhnliches, sondern so sollte es sein. In einer solchen Situation wird die Frage „Wer bestimmt die Redaktionsgrundsätze einer rom Westen finanzierten Kirchenzeitung? " zu einem wirklichen Problem - leider.

Wenn auch die Kirchenpresse als ein Instrument der nationalen Entwicklung ihren Beitrag leisten muß, heißt das doch nicht, daß sie ihre Unabhängigkeit verlieren darf. Unabhängigkeit in diesem Sinne heißt nicht Uberheblichkeit, sondern Widerstand gegen willkürlichen Druck, wenn solcher Druck die besten Werte und das Gemeinwohl des Landes behindert. Glücklicherweise halten einige vorausschauende Länder Afrikas nichts von Pressekontrolle. So sagte etwa Sambias Minister für Information, Rundfunk und Tourismus, Sikota Wina, bei einer Studentengraduation des Africa Literature Centre im Mai 1971:

„Eine Volksregierung ohne eine freie Volkspresse ist der Anfang einer Farce oder einer Tragödie. Journalisten müssen die anerkannten Leitsätze ihres Berufs respektieren ... Sie sind ... wahrscheinlich von größter Bedeutung und Wirkung in einer Zeit, wo das geschriebene Wort mehr Macht hat als der Gesetzgeber."

Das folgende Zitat stammt von einem lateinamerikanischen Herausgeber, Dr. Gainze Paz von "La Prensa" (Buenos Aires):

"Wir wissen um die Verantwortung jedes guten Redakteurs, das Interesse seiner Leser für den Fortschritt und die Entwicklung ihres Landes zu fördern. Wir wissen auch, daß wir Wege finden müssen, dieses Interesse zu wecken, obwohl nichtinformierte Leser manchmal in der Tat indifferent zu sein scheinen. Wir wissen auch, daß Zeitungen Nachrichten ohne Verdrehungen, ohne Furcht vor der Veröffentlichung widersprechender Standpunkte, interpretieren sollten. Und, was vor allem wichtig ist: eine Zeitung muß ihre eigene Meinung klar und ohne Rücksicht auf die Folgen zum Ausdruck bringen. Uber die Pflicht zu informieren, über redaktionelle Richtlinien, so mutig sie auch sein mögen, hinaus hat der wahre Journalist die Aufgabe, zwischen den Lesern und der Zeitung ein Band des Vertrauens zu schaffen. Nur so kann er auf die Unterstützung der Leser hoffen, wenn es darum geht, eine Pressefreiheit zu verteidigen, die das Informationsrecht der Bevölkerung garantiert."

Wer entscheidet nun aber bei einer kirchlichen Zeitung über die redaktionellen Richtlinien? Das hängt von der Organisationsstruktur des Periodikums ab. Manchmal setzen sie bei den Bischöfen an, bei der Bischofskonferenz oder dem höchsten Gremium der Kirche - der Synode, einer Konferenz oder dem Presbyterium. Diese delegieren die Verantwortung gewöhnlich an eine untergeordnete Gruppe wie einen Kommunikationsausschuß, dem Mitglieder aus verschiedenen Organisationen der Kirche angehören. Dieser ernennt den Redakteur, gewöhnlich mit Zustimmung oder 
Einwilligung der Bischofskonferenz. Er ernennt auch ein kleines Redaktionskomitee, das ihn berät und kritisch begleitet.

Leider werden die Grundsätze für die Redaktionsarbeit allzu häufig von oben nach unten festgelegt wie auf einer Einbahnstraße. Das ist dann der Grund dafür, daß solche Blätter niemanden ansprechen als die geheiligten Kirchenwände. Ein wirkungs: vollerer Weg bei der Erstellung solcher Grundsätze ist es, die Wunschbeiträge von Lesern oder potentiellen Lesern einschließlich der kritischen Leute zu integrieren.

Das kann durch Fragebögen oder mit Briefen an die Redaktion geschehen. Die Leser werden auf diese Weise dahin gelangen, die Zeitung "ihre Zeitung“ zu nennen.

\section{S U M M A R Y}

The autor endeavours to present a clear editorial draft, editorial principles and guidelines for today's religious press in Africa. At this time, the religious press in Africa should not be content to rank second to a secular press, which is becoming stronger. It should always be the voice of conscience and of human needs. The autor does not offer any solution, but provokes some questions which have to be raised the more the press has to find their way between the demands of western values and the rising demands of African values. He warns them to fix the principles for editorial work of the religious press of today's Africa, like on a one-way road, from top to bottom.

\section{RESUME}

Ce qui importe à l'auteur, c'est un clair concept de rédaction; plus exactement, se sont les instructions et les contrastes rédactionnels de la presse spirituelle, en Afrique, aujourd'hui. Tout d'abord, la presse spirituelle en Afrique n'a pas besoin de se contenter d'une deuxième place à côté de la presse séculaire en forte progression. Elle restera toujours la voix de la conscience et du souci humain. L'auteur n'offre pas de solutions, mais pose seulement, de façon consciente, les questions qui sont à poser, d'autant que la presse doit, en général, trouver son chemin entre l'exigence de puissance des valeurs occidentales et l'exigence nouvellement née des valeurs africaines. Il met en garde afin qu'on ne fixe pas les principes pour le travail rédactionnel de la presse spirituelle dans l'Afrique actuelle, de haut en bas, comme dans une rue à sens unique.

\section{RESUMEN}

El autor se interesa por un concepto redaccional claro, más que de directrices redaccionales o de principios para la prensa eclesiástica en el Africa de hoy. En primer lugar la prensa eclesiástica en Africa no necesita contentarse con un segundo puesto junto a la prensa secular, de más amplia difusión. Ella será siempre la voz de la conciencia y de la inquietud humana. El autor no ofrece soluciones, simplemente plantea conscientemente los interrogantes que es necessario plantearse mientras la prensa busca su propio camino entre las pretensiones de los valores occidentales y las nuevas y crecientes reivindicaciones de los valores africanos. Advierte de los peligros de fijar desde arriba una dirección única para el trabajo redaccional de la prensa eclesiástica en el Africa de hoy. 